\title{
Coordinated Expression of 6-Phosphofructo-2-kinase/ Fructose-2,6-bisphosphatase 4 and Heme Oxygenase 2: Evidence for a Regulatory Link between Glycolysis and Heme Catabolism
}

\author{
Bin Li, ${ }^{1}$ Kazuhisa Takeda, ${ }^{1}$ Kazunobu Ishikawa, ${ }^{2}$ Miki Yoshizawa, ${ }^{1}$ \\ Michihiko Sato, ${ }^{3}$ Shigeki Shibahara ${ }^{1}$ and Kazumichi Furuyama ${ }^{1}$ \\ ${ }^{1}$ Department of Molecular Biology and Applied Physiology, Tohoku University School of Medicine, Sendai, Japan
${ }^{2}$ Center for Medical Education and Career Development, Fukushima Medical University, Fukushima, Japan
${ }^{3}$ Central Laboratory for Research and Education, Yamagata University School of Medicine, Yamagata, Japan
}

Heme is an essential requirement for cell survival. Heme oxygenase $(\mathrm{HO})$ is the rate-limiting enzyme in heme catabolism and consists of two isozymes, HO-1 and HO-2. To identify the protein that regulates the expression or function of $\mathrm{HO}-1$ or $\mathrm{HO}-2$, we searched for proteins that interact with both isozymes, using protein microarrays. We thus identified 6-phosphofructo-2-kinase/fructose-2,6-bisphosphatase 4 (PFKFB4) that synthesizes or degrades fructose-2,6-bisphosphate, a key activator of glycolysis, depending on cellular microenvironments. Importantly, HO-2 and PFKFB4 are predominantly expressed in haploid spermatids. Here, we show a drastic reduction in expression levels of PFKFB4 mRNA and protein and HO-2 mRNA in HepG2 human hepatoma cells in responses to glucose deprivation $(\leq 2.5 \mathrm{mM})$, which occurred concurrently with remarkable induction of HO-1 mRNA and protein. Knockdown of HO-2 expression in HepG2 cells, using small interfering RNA, caused PFKFB4 mRNA levels to decrease with a concurrent increase in HO-1 expression. Thus, in HepG2 cells, HO-1 expression was increased, when expression levels of HO-2 and PFKFB4 mRNAs were decreased. Conversely, overexpression of HO-2 in HepG2 cells caused the level of co-expressed PFKFB4 protein to increase. These results suggest a potential regulatory role for HO-2 in ensuring PFKFB4 expression. Moreover, in D407 human retinal pigment epithelial cells, glucose deprivation decreased the expression levels of PFKFB4, HO-1, and HO-2 mRNAs. Thus, glucose deprivation consistently down-regulated the expression of PFKFB4 and HO-2 mRNAs in both HepG2 cells and RPE cells. We therefore postulate that PFKFB4 and HO-2 are expressed in a coordinated manner to maintain glucose homeostasis.

Keywords: diabetes mellitus; glucose; glycolysis; liver; retinal pigment epithelium Tohoku J. Exp. Med., 2012 Sep, 228 (1), 27-41. C 2012 Tohoku University Medical Press

Heme is an essential requirement for cell survival (Furuyama et al. 2007). Heme is cleaved by the microsomal heme oxygenase (HO) system to release iron, carbon monoxide and biliverdin at the expense of molecular oxygen and NADPH (Tenhunen et al. 1968; Yoshida and Kikuchi 1978). HO consists of two structurally related isozymes, HO-1 and HO-2 (Shibahara et al. 1985; Maines et al. 1986; Ishikawa et al. 1995), both of which catalyze the same reaction (Shibahara et al. 1993; Ishikawa et al. 1995). Indeed, the catalytic domains of human HO-1 and HO-2 share about $93 \%$ amino-acid sequence identity (Yoshida et al. 1988; Ishikawa et al. 1995), while the overall aminoacid sequence similarity is about $43 \%$. Importantly, HO-2 may function as a hemoprotein by binding heme through the heme regulatory motifs, each containing a Cys-Pro dipeptide (McCoubrey et al. 1997). It is therefore conceivable that HO-2 may possess a hitherto unknown function in regulating cellular homeostasis. Moreover, the expression level of HO-2 is maintained in narrow ranges in various human cell types (Takahashi et al. 1996; Shibahara et al. 2007; Li et al. 2008), the property of which may be suitable for its regulatory roles. In contrast, expression of HO- 1 is induced (Takeda et al. 1994; Takahashi et al. 1996; Nakayama et al. 2000) or reduced under various conditions (Takahashi et al. 1999; Kitamuro et al. 2003; UdonoFujimori et al. 2004; Shibahara et al. 2007). Moreover, the human HO-1 gene is under the transcriptional regulation that may vary depending on the lengths of GT repeats

Received June 28, 2012; accepted July 31, 2012. Published online August 15, 2012; doi: 10.1620/tjem.228.27.

Correspondence: Kazuhisa Takeda, M.D., Ph.D., Department of Molecular Biology and Applied Physiology, Tohoku University School of Medicine, 2-1 Seiryo-machi, Aoba-ku, Sendai, Miyagi 980-8575, Japan.

e-mail: ktakeda@med.tohoku.ac.jp 
located in its promoter region (Okinaga et al. 1996; Kimpara et al. 1997; Yamada et al. 2000; Shibahara 2003); namely, the length polymorphism of the GT repeats may influence the expression level of the HO-1 gene (Hirai et al. 2003), which may account in part for the inter-individual difference.

HO-2-deficient mice (HO- $2^{-/-}$mice) are fertile and show relatively mild phenotypes (Poss et al. 1995), compared to the critical conditions of HO-1-deficient mice (Poss and Tonegawa 1997). HO- $2^{-/-}$mice are characterized by the attenuated hypoxic ventilatory responses with normal hypercapnic ventilatory responses (Adachi et al. 2004). We have recently reported that the expression level of HO-1 protein is lower by $35 \%$ in the $\mathrm{HO}-2^{-/-}$mouse liver compared to that in wild-type mouse liver (Han et al. 2010), whereas it was higher in the testis and heart of the $\mathrm{HO}-2^{-/-}$ mouse (Han et al. 2009). In addition, other investigators reported that the HO-1 protein level was lower by $40 \%$ in the HO- $2^{-/-}$mouse kidney (Sodhi et al. 2009). The decrease in the expression levels of HO-1 protein in the liver and kidney of $\mathrm{HO}-2^{-/-}$mouse is of particular interest, because these two organs are characterized by their capability to release glucose to the systemic circulation, the activity of which is enhanced in diabetic patients (Meyer et al. 1998). In fact, $\mathrm{HO}-2^{-/-}$mice exhibit hyperglycemia and insulin resistance (Sodhi et al. 2009), thereby suggesting that HO-2 deficiency may disturb the balance between glycolysis and gluconeogenesis. It is therefore conceivable that HO-2 may possess hitherto unknown functions in oxygen sensing and glucose homeostasis.

In the present study, using protein microarrays, we identified several candidate proteins that may interact with both HO-1 and HO-2 and presumably are involved in regulating the function of HO-1 and/or HO-2. Among these potential binding proteins, we are interested in 6-phosphofructo-2-kinase/fructose-2,6-bisphosphatase 4 (PFKFB4), because PFKFB4 is a bifunctional enzyme that synthesizes or degrades fructose-2,6-bisphosphate, a key activator of glycolysis (Okar et al. 2001). Among the four PFKFB isozymes that have been reported, PFKFB4 exhibits a relatively high kinase/phosphatase activity ratio (Sakata et al. 1991). Recently, PFKFB4 has been shown to ensure the survival of prostate cancer cells (Ros et al. 2012), glioblastoma (Goidts et al. 2012), and hepatocellular carcinoma cells (Jeon et al. 2011). In this context, our group reported higher expression levels of HO-1 and HO-2 mRNAs in glioma specimens, including anaplastic astrocytoma and glioblastoma multiforme, compared to the normal brain tissue (Hara et al. 1996). The high expression levels of HO-1 and HO-2 may lead to the supply of sufficient amounts of iron, an essential requirement for cell proliferation, along with bilirubin, a potent radical scavenger (Shibahara 2003). These heme degradation products are beneficial for cell proliferation or survival of glioblastoma cells.

Here, we provide several lines of evidence for the coordinated expression of PFKFB4 and HO-2, utilizing
$\mathrm{HO}-2^{-/-}$mice and human cell lines with distinctive metabolic and physiologic properties.

\section{Materials and Methods}

\section{Preparation of recombinant $\mathrm{HO}-1$ and $\mathrm{HO}-2$ proteins}

The HO-1 cDNA segment (position 81-878) and the HO-2 cDNA segment (position 85-939), each carrying the protein-coding region without the $\mathrm{C}$-terminal hydrophobic trans-membrane domain (TMD), were amplified by PCR from human HO-1 cDNA pHHO1 (Yoshida et al. 1988) (GenBank accession number X06985) and human HO-2 cDNA (Shibahara et al. 1993) (GenBank accession number X002134), respectively. Each of the amplified fragments was subcloned into a prokaryote expression vector, pIVEX 2.4d (Roche, Diagnostics), yielding pIVEX-HO-1 and pIVEX-HO-2. The validity of every construct used in the present study was confirmed by DNA sequencing. The pIVEX constructs were used for in vitro translation system derived from Escherichia (E.) coli (Rapid Translation System 500 E. coli HY Kit, Roche). The HO-1 and HO-2 proteins, lacking the $\mathrm{C}$-terminal TMD, were produced as hexa-histidine tagged proteins from pIVEX-HO-1 and pIVEX-HO-2, respectively. The produced HO-1 and HO-2 proteins were purified with immobilized $\mathrm{Ni}^{2+}$ beads. The proteins were bound to nickel resin with binding buffer, which contained $5 \mathrm{mM}$ imidazole, $0.5 \mathrm{M} \mathrm{NaCl}$, and $20 \mathrm{mM}$ Tris- $\mathrm{HCl} \mathrm{pH} 7.9$. The contaminated proteins were removed with washing buffer that contained $30 \mathrm{mM}$ imidazole. HO-1 and HO-2 proteins were eluted with elution buffer containing $1 \mathrm{M}$ imidazole. After dialysis against PBS, the purified HO-1 and HO-2 proteins were concentrated with centricon centrifugal filter (Millipore) at 7,000 $\times \mathrm{g}$ for $2 \mathrm{~h}$.

\section{Protein microarrays}

ProtoArray ${ }^{\mathrm{TM}}$ Human Protein Microarray PPI Kits (Invitrogen, Carlsbad, California) were used following the manufacture's protocol. The protein microarrays contain a total of $\sim 1,900$ species of purified tagged proteins. Every human protein was produced as a fusion protein containing glutathione-S-transferase at its $\mathrm{N}$-terminal and was fixed in duplicate on a nitrocellulose-coated glass slide (the microarray). The purified 6xHis-tagged HO-1 or HO-2 protein was biotinylated as a probe, each of which was then incubated with the protein microarray. The slides were blocked in $1 \%$ bovine serum albumin (BSA) in phosphate-buffered saline (PBS) containing $0.1 \%$ Tween-20 on ice for $1 \mathrm{~h}$ at cold room. The biotinylated HO-1 or HO-2 protein was diluted with $120 \mu \mathrm{l}$ probing buffer $(50 \mu \mathrm{g} / \mathrm{ml})$ and loaded on the slides. The slide was covered with a HybriSlip ${ }^{\mathrm{TM}}$ Cover Slip (Invitrogen). The slides were then placed in a 50-ml conical tube and incubated for $1.5 \mathrm{~h}$ at $4^{\circ} \mathrm{C}$. Afterwards, the slides were washed with probing buffer and incubated with Streptavidin-Alexa Fluor 647 Conjugate on ice in the dark room for $30 \mathrm{~min}$. After washing and drying, the slides were scanned with a fluorescence microarray scanner, GenePix Pro 4.0 (Axon Instruments, Inc.).

\section{Co-immunoprecipitation of HO-1 or HO-2 with PFKFB4 protein}

The full-length HO-1 or HO-2 cDNA was ligated to pUB6/ V5-His mammalian expression vector (Invitrogen, Carlsbad, CA), yielding pUB6-HO-1 or pUB6-HO-2. The expression vector, pMycPFKFB4, for the entire PFKFB4 protein fused with Myc-tag at the C-terminus was constructed as follows. The PCR-amplified PFKFB4 cDNA fragment carrying the HindIII site at its $5^{\prime}$-end and the SalI site at its $3^{\prime}$-end was cloned between the HindIII and SalI sites of pMycN1 (Yasumoto et al. 2002), yielding pMyc-PFKFB4. The expression 
vector pMyc-N1 was constructed by replacing the enhanced green fluorescent protein (EGFP) cDNA of pEGFP-N1 (clontech) with a c-Myc-tag (GAG CAG AAA CTC ATC TCA GGG GAG GAT CTG TAG); namely, pMyc-N1 carries the c-Myc tag between the NcoI site and the NotI site downstream from the Cytomegalovirus (CMV) promoter (Yasumoto et al. 2002). The validity of each construct was confirmed by DNA sequencing.

COS7 monkey kidney cells were maintained in Dulbecco's Modified Eagle's Medium (DMEM), supplemented with $10 \%$ fetal bovine serum (FBS) and antibiotics $(100 \mathrm{U} / \mathrm{ml}$ penicillin and $0.1 \mathrm{mg} /$ $\mathrm{ml}$ streptomycin), and transfected with pUB6-HO-1 or pUB6-HO-2, and pMyc-PFKFB4. After $48 \mathrm{~h}$, cells were lysed with $50 \mathrm{mM}$ Tris$\mathrm{HCl} \mathrm{pH} 7.5,150 \mathrm{mM} \mathrm{NaCl}, 1 \% \mathrm{NP}-40,1 \mathrm{mM}$ EDTA and 1\% protease inhibitor cocktail, and the lysates were incubated with Protein G Sepharose at $4{ }^{\circ} \mathrm{C}$ with gentle rotation for $2 \mathrm{~h}$ and centrifuged. The lysis buffer used did not contain a phosphatase inhibitor, because at this stage, we did not intend to measure the phosphorylated PFKFB4. The supernatant was transferred to a new tube mixed with anti-V5 antibody and protein $\mathrm{G}$ Sepharose and rotated at $4{ }^{\circ} \mathrm{C}$ overnight. The protein $\mathrm{G}$ Sepharose was then washed by the lysis buffer and eluted with $0.1 \mathrm{M}$ glycine- $\mathrm{HCl}$ buffer, $\mathrm{pH} 3.5$. Proteins were analyzed on a $12 \%$ SDS-PAGE, and detected with anti-Myc antibody (Santa Cruz CA, USA) and anti-V5 antibody (Invitrogen). Immunoreactive proteins were detected with a Western blot kit (Millipore Corporation).

\section{Pull-down assay}

The HO-1 cDNA segment (position 81-878) and the HO-2 cDNA segment (position 85-939), each carrying the protein-coding region without the $\mathrm{C}$-terminal TMD, were cloned into a prokaryote expression vector, pET-28a (Novagen, Darmstadt, Germany), yielding pET-28a-HO-1 and pET-28a-HO-2, respectively. The expression vector for the entire PFKFB4 protein fused with hemagglutinin (HA)tag at the C-terminus was constructed as pHA-PFKFB4. E. coli BL21 (DE3) cells (Invitrogen) were transformed with pET-28a-HO-1, pET-28a-HO-2 or pET-28a (as a negative control) for generation of hexa-histidine tagged HO-1 or HO-2 protein. The transformed BL21 (DE3) cells were sonicated, and centrifuged. Then, the supernatant was mixed with $\mathrm{Ni}^{2+}$ resin (Qiagen, Valencia, CA) at $4{ }^{\circ} \mathrm{C}$ with gently rotating for $2 \mathrm{~h}$. Thereafter the $\mathrm{Ni}^{2+}$ resin was washed, incubated with the whole cell extracts of COS7 cells transfected with pHA-PFKFB4, and washed again. After that, the hexa-histidine tagged HO-1 or HO-2 was eluted with elution buffer, and subjected to the western blot analysis with anti-HA antibody (Sigma-Aldrich, St. Louis, MO, USA) or anti-His-tag antibody (Santa Cruz CA, USA).

\section{Animal experiments}

Animal experiments were performed according to the institutionally approved protocol of Fukushima Medical University and that of Tohoku University School of Medicine. HO-2 $2^{-/}$mice with $\mathrm{C} 57 \mathrm{BL} / 6 \mathrm{~J} \times 129 / \mathrm{Sv}$ mixed genetic background were generated as previously described (Poss et al. 1995; Adachi et al. 2004) and maintained in Fukushima Medical University. Body weight and the testis weight were measured in male $\mathrm{HO}-2^{-/-}$mice (12 weeks old) and male age-matched wild-type $\mathrm{C} 57 \mathrm{BL} / 6 \mathrm{~J}$ mice. Tissue extracts were prepared from the testis and liver of $\mathrm{HO}_{-} 2^{-/-}$mice (10 weeks old) and age-matched wild-type mice, as described previously (Han et al. 2009, 2010).

\section{Effects of glucose deprivation}

HepG2 human hepatoma cells and D407 human retinal pigment epithelial (RPE) cells (Davis et al. 1995) were maintained in Dulbecco's Modified Eagle's Medium (DMEM) containing high glucose (25 mM), supplemented with 10\% FBS and antibiotics (100 U/ $\mathrm{ml}$ penicillin and $0.1 \mathrm{mg} / \mathrm{ml}$ streptomycin). ARPE-19 cells (Dunn et al. 1996) were obtained from Leonard M. Hjelmeland (University of California, Davis, CA, USA). ARPE-19 cells were cultured in a 1:1 mixture of DMEM and Ham's F12, supplemented with 10\% FBS and antibiotics. Glucose-free DMEM was supplemented with $1 \%$ FBS and antibiotics. Cells were cultured in $10-\mathrm{cm}$ dishes to $70-80 \%$ confluence, rinsed two times with PBS, and then incubated for $12 \mathrm{~h}$ in DMEM with glucose concentration from 0 to $25 \mathrm{mM}$. It should be noted that each culture medium contained a small amount of glucose derived from $1 \%$ FBS $(\sim 0.05 \mathrm{mM}$ glucose).

Northern blot analysis was performed with the DIG Northern Starter Kit (Roche Diagnostics Japan). The HO-1 and HO-2 RNA probes were prepared, as described previously (Zhang et al. 2006). For preparation of PFKFB4 RNA probe, the human PFKFB4 cDNA of positions 450-1430 (GenBank accession number NM 004567) was amplified by PCR, and then cloned into pGEM-Teasy vector (Promega, Madison, WI), named pGEM-PFKFB4. The cDNA segment for non-specific 5-aminolevulinate synthase (ALAS-N) was amplified by PCR from human placenta cDNA (forward primer: CTCAGCGCAGTCTTTCCACAGG and reverse primer: TAATTC ACATATAAAGACAACTC) and cloned into pGEM-Teasy, named pGEM-Teasy-ALAS-N. SP6 RNA polymerase was used for transcription of each RNA probe.

Western blot analysis for HO-1 protein was performed, as detailed previously (Zhang et al. 2006; Ding et al. 2006). The antibodies used were anti-HO-1 antibody (Shibahara et al. 1993) at a dilution of 1:1,000 and anti- $\beta$-actin antibody (Sigma-Aldrich, St. Louis, MO) at a dilution of 1:5,000. For detection of HO-2 protein, the blot was incubated overnight at $4{ }^{\circ} \mathrm{C}$ with $\mathrm{HO}-2$ antibody (Stressgen, Victoria, BC, Canada) at a dilution of 1:2,000.

\section{Knockdown of HO mRNA expression}

The small interfering RNA (siRNA) against HO-2 mRNA (target base 248-272), named siHO-2, and scrambled HO-2 siRNA (negative control) were used, as described previously (Ding et al. 2006). Likewise, a specific siRNA against HO-1, siHO-1 (Miralem et al. 2005), and glyceraldehyde-3-phosphate dehydrogenase (GAPDH) siRNA, siGAPDH (a control for siRNA treatment), were used (Ding et al. 2006). HepG2 cells were cultured in 6-well plates in DMEM containing high glucose $(25 \mathrm{mM})$, supplemented with $10 \%$ FBS, and were treated with each siRNA (6 pmoles) and Lipofectamine RNAiMAX (Invitrogen, Carlsbad, CA) according to a manufacturer's protocol. HepG2 cells were also treated with Lipofectamine RNAiMAX alone as a control. After $36 \mathrm{~h}$, the cells were harvested for RNA extraction.

\section{Transient co-expression of HO-1 or HO-2 with PFKFB4}

The full-length HO-1 cDNA was cloned in the expression vector pRc/CMV (Invitrogen, Carlsbad, CA), yielding pRc/CMV-HO-1 (Ding et al. 2006). The entire protein-coding region of HO-2 was amplified by PCR and inserted into the expression vector pcDNA3 (Invitrogen), yielding pcDNA3-HO-2. The expression vector pMycPFKFB4 codes for human PFKFB4 fused with the c-Myc tag at the $\mathrm{C}$-terminus, as described above. Accordingly, every expression vec- 
tor used in co-transfection assays carries each cDNA under the control of the CMV promoter.

HepG2 cells in a 6-cm culture dish were transfected with $\mathrm{pRc} /$ CMV-HO-1, pcDNA3-HO-2, or pcDNA3 (empty vector), together with pMyc-PFKFB4 or vector DNA using FuGENE HD (Roche, Mannheim, Germany), and incubated for $48 \mathrm{~h}$. The amount of DNA used was $2.5 \mu \mathrm{g}$ for each plasmid. Cell lysates were prepared and were subjected to the Western blot analysis (20 $\mu$ g protein per lane), as described previously ( $\mathrm{Li}$ et al. 2008). The antibody preparations used were anti-HO-1 antibody, anti-HO-2 antibody, anti-PFKFB4 antibody (ABGENT, San Diego, CA), anti-neomycin phosphotransferase-II antibody (Upstate, Temecula, CA), and anti- $\beta$-actin antibody. Anti-neomycin phosphotransferase-II antibody was used for evaluating a transfection efficiency, because neomycin phosphotransferase II gene was expressed under the control of SV40 promoter. The neomycin phosphotransferase II gene with the SV40 promoter is present in all expression vectors ( $\mathrm{HO}$ and PFKFB4 expression vectors used for transfection).

HeLa human cervical cancer cells were cultured in Minimum Essential Medium Eagle, supplemented with 10\% FBS and antibiotics. HeLa cells, $\sim 90 \%$ confluent in a $15-\mathrm{cm}$ culture dish, were transfected with pRc/CMV-HO-1, pcDNA3-HO-2, or pcDNA3 (empty vector), together with pMyc-PFKFB4 or vector DNA using FuGENE $\mathrm{HD}$ for $24 \mathrm{~h}$ and harvested. Transfected cells were used for western blot analysis (10\% of transfected cells) and for HO enzyme assay ( $90 \%$ of transfected cells), because a large number of cells were required for preparation of the microsomal fraction. The amount of DNA used was $15 \mu \mathrm{g}$ for each plasmid. The harvested HeLa cells were used for the western blot analysis ( $\mathrm{Li}$ et al. 2008). Anti- $\alpha$ tubulin antibodies were obtained from Lab Vision (Fremont, CA) and expression level of $\alpha$-tubulin served as a loading control measure. Anti-Myc antibody (Santa Cruz, CA) was also used to confirm the expression of PFKFB4. The blots were incubated overnight at $4^{\circ} \mathrm{C}$ with a respective antibody at a dilution of $1: 1000$.

\section{Assay for HO catalytic activity}

The transfected HeLa cells were used for measuring the HO activity in microsomal preparations (Shibahara et al. 1978). Each sample (100 $\mu \mathrm{g}$ microsomal proteins) was added to $200 \mu \mathrm{l}$ of the reaction mixture, which contained 0.1M KPB (pH 7.4), $15 \mu \mathrm{M}$ hemin, $100 \mu \mathrm{g} / \mathrm{ml}$ bovine serum albumin, and $40 \mu \mathrm{g}$ recombinant human biliverdin reductase (Sun et al. 2000). Heme oxygenase activity was expressed as nmole bilirubin/mg protein $/ \mathrm{h}$.

\section{Statistical analysis}

Data are presented as mean \pm standard deviation (s.D.). Comparison between two groups was performed with the Student's $t$-test. Differences between mean values were considered significant when $p<0.05$.

\section{Results and Discussion}

PFKFB4 as a potential binding protein for $\mathrm{HO}-1$ and $\mathrm{HO}-2$

To identify the proteins that regulate the function or expression of HO-1 and/or HO-2, we searched for the proteins that interact with both enzymes using the protein
A

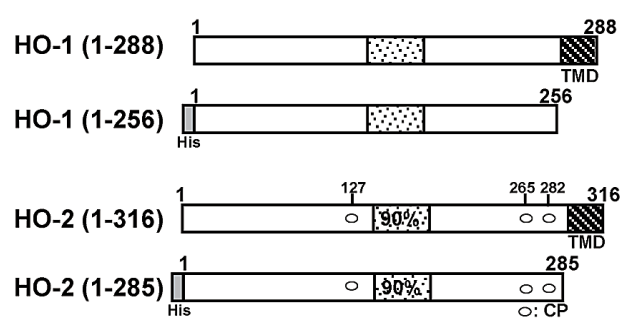

B

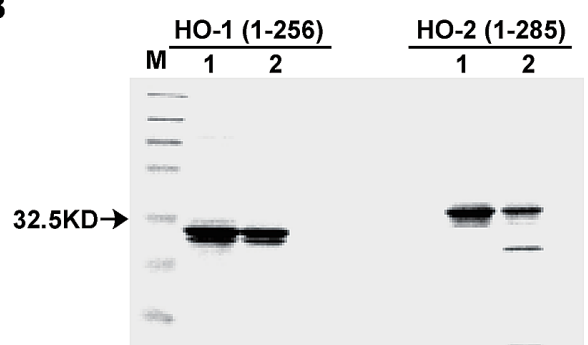

C
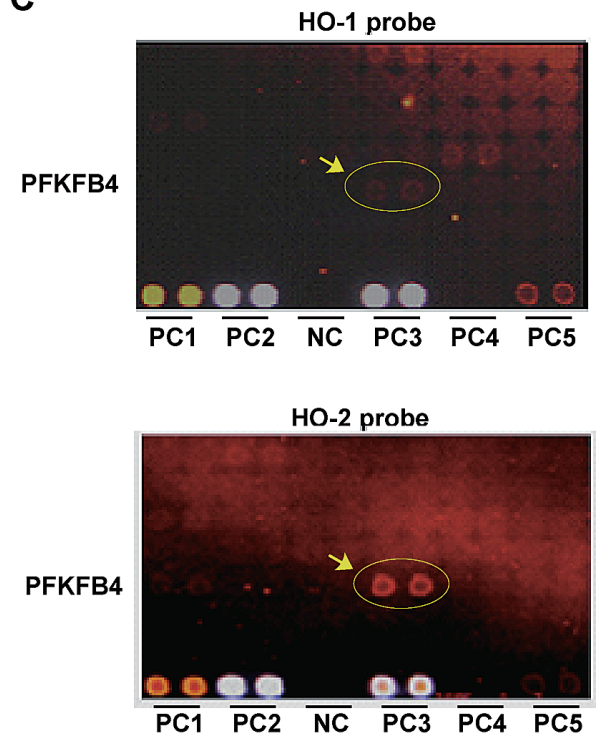

Fig. 1. Protein microarray assay for interaction between PFKFB4 and HO-1 or HO-2.

(A) Schematic diagram of HO-1 and HO-2 constructs. Truncated HO-1 (1-256) and HO-2 (1-285) lack TMD. The conserved catalytic domain is shown as stipple, and the number indicates the amino acid sequence identity to human HO- 1 . Human HO-2 contains three copies of CP motif, and the position numbers of Cys residues are shown. (B) Purified HO-1 and HO-2 protein without TMD. The produced HO-1 and HO-2 proteins were analyzed by staining SDS-polyacrylamide gel with Coomassie brilliant blue. Lanes 1 and 2 contain two independent preparations of purified HO-1 and HO-2 proteins. (C) Images of protein microarrays. Biotinylated HO-1 or HO-2 protein was subjected to the protein microarrays. Every human protein is spotted on the microarray in duplicate. The positive signals (PFKFB4) are indicated with an arrow. Positive controls, PC1 and PC2, contain the biotin Ab gradient to detect biotinylated probes; PC3, antibiotin $\mathrm{Ab}$ for detecting biotinylated probes; and PC4 and PC5, the V5 control that interacts with the Streptavidin-Alexa Fluor 647 Conjugate. Negative control (NC) was spotted with buffer only. 
Table 1. The list of proteins that bind to HO-1 and HO-2 on the microarrays.

\begin{tabular}{ll}
\hline Gene name & \\
\hline MPG & N-methylpurine-DNA glycosylase \\
HMGN1 & High-mobility group nucleosome binding domain 1 \\
CSNK1G1 & Casein kinase 1, gamma 1 \\
STK6 & Serine/threonine kinase 6 \\
EIF2S2 & Eukaryotic translation initiation factor 2, subunit 2 beta, 38 kDa \\
PCCA & Propionyl Coenzyme A carboxylase, alpha polypeptide \\
LCP2 & Lymphocyte cytosolic protein 2 (SH2 domain containing leukocyte protein of 76 kDa) \\
METAP2 & Methionyl aminopeptidase 2 \\
DDA3 & Differential display and activated by p53 \\
PFKFB4 & 6-phosphofructo-2-kinase/fructose-2,6-biphosphatase 4 \\
\hline
\end{tabular}

Shown are the proteins that gave strong signals on the microarrays with biotinylated HO-1 and HO-2 proteins.

microarrays. The probes used were biotinylated HO-1 protein and biotinylated HO-2 protein, each of which lacks a carboxyl-terminal TMD (Fig. 1A, B). TMD is required for the membrane insertion, but is dispensable for the catalytic function (Yoshida and Sato 1989; Yoshida et al. 1991). We thus identified several candidate proteins, some of which are listed in Table 1. Among these candidate proteins, we are particularly interested in PFKFB4, because $\mathrm{HO}-2^{-/-}$ mice manifest hyperglycemia and insulin resistance (Sodhi et al. 2009), the phenotype of which suggests the potential involvement of HO-2 in glucose homeostasis. Accordingly, the images of the microarray are shown after probing with biotinylated HO-1 or HO-2 protein (Fig. 1C). The strong signals were detected on the spots of PFKFB4 with the HO-2 probe, compared to the faint signals with the HO-1 probe. These results suggest that HO-2 may bind PFKFB4 and that the interaction between HO-2 and PFKFB4 may be stronger than that between HO-1 and PFKFB4.

PFKFB4 functions as a homodimer through the interaction at the N-terminal (Okar et al. 2001). The N-terminal half of PFKFB4 functions as a kinase, while the C-terminal half functions as a bisphosphatase. The catalytic activity of PFKFB4 is regulated by phosphorylation and dephsophorylation. In case of the protein microarray, however, PFKFB4 protein was fixed to a glass plate as a monomer tagged with glutathione-S-transferase at the $\mathrm{N}$-terminus. It is therefore conceivable that either HO-1 or HO-2 probe may bind a monomer of PFKFB4 protein. It should be noted that HO-1 and HO-2 are anchored to the endoplasmic reticulum through a respective carboxyl-terminal TMD of about 30 amino acids (Yoshida and Sato 1989; Yoshida et al. 1991). Accordingly, the amino-terminal large portion of each HO isozyme is present in the cytoplasm (see Fig. 8), where PFKFB4 is also present (Yalcin et al. 2009).

\section{In vitro interaction between $\mathrm{PFKFB} 4$ and $\mathrm{HO}-1$ or $\mathrm{HO}-2$}

To confirm the interaction detected on the protein microarray between PFKFB4 and HO-1 or HO-2, we carried out immunoprecipitation assays by expressing Myc- tagged PFKFB4 and V5-tagged full-length HO-1 or HO-2 in COS7 monkey kidney cells (Fig. 2A). The input of the Myc-tagged PFKFB4 protein was detected with anti-Myc antibody in the samples of cell extracts, indicating the successful expression of PFKFB4 protein in transfected cells. PFKFB4 protein that was co-precipitated with HO-1 or HO-2 protein was then detected with anti-Myc antibody, whereas only faint band of background was detected in the whole cell extracts prepared from COS7 cells transfected with empty vector (Fig. 2A). The V5-tagged HO-1 and HO-2 proteins were detected with anti-V5 antibody in the re-probed membrane, but not in the whole cell extracts prepared from mock transfected cells.

We next performed pull-down assays with hexa-histidine to further verify the interaction between PFKFB4 and HO-1 or HO-2 (Fig. 2B). The whole cell extracts of COS7 cells, which express HA-tagged PFKFB4 protein, were incubated with the $\mathrm{Ni}^{2+}$ resin that was bound by the hexahistidine-tagged HO-1 or HO-2 protein lacking TMD. Subsequently, the hexa-histidine-tagged HO-1 or HO-2 protein was eluted from the $\mathrm{Ni}^{2+}$ resin. PFKFB4 protein was eluted together with HO-1 or HO-2 protein, but PFKFB4 protein was not eluted in the case of empty vector. All these results support the in vitro interaction between PFKFB4 and HO-1 or HO-2.

We also confirmed the colocalization of tagged HO-1 or HO-2 with tagged PFKFB4 in COS7 cells (data not shown) and the interaction between HO-2 and PFKFB4 by mammalian two-hybrid assays (data not shown). However, we were unable to confirm the interaction between the endogenous proteins. We therefore performed various series of experiments to explore the regulatory link between PFKFB4 and HO-1 or HO-2.

The liver-specific regulation of HO-1 expression in the $\mathrm{HO}-2^{-/}$mouse

We initially analyzed the expression of PFKFB4 and HO-1 proteins in $\mathrm{HO}-2^{-/-}$mice that manifest hyperglycemia and insulin resistance (Sodhi et al. 2009). We confirmed 
A
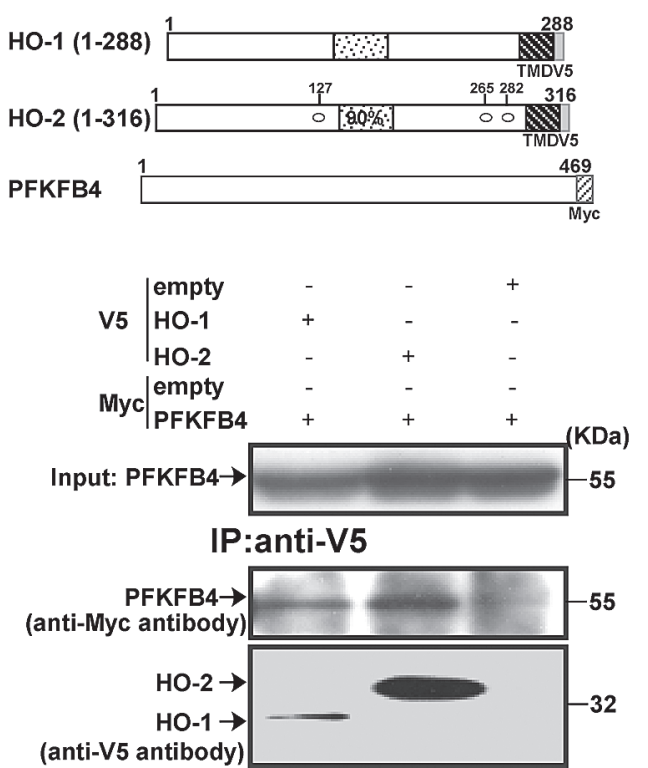

B
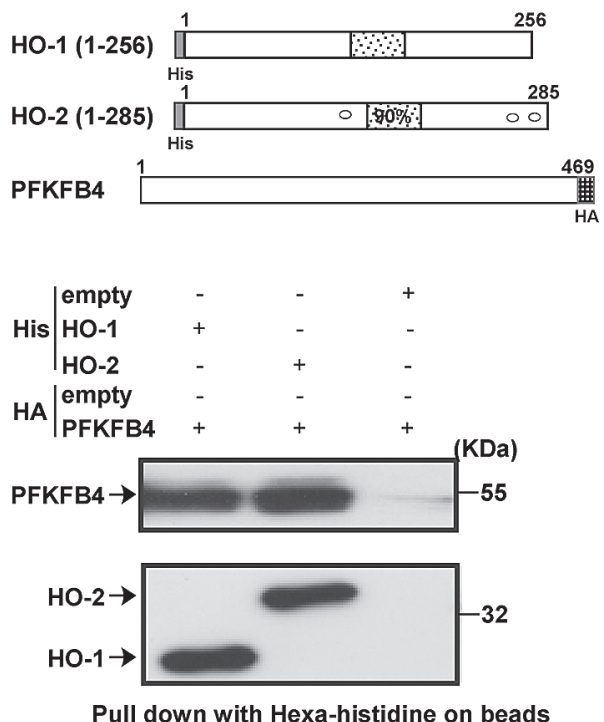

Pull down with Hexa-histidine on beads

Fig. 2. In vitro interaction between PFKFB4 and HO-1 or HO-2.

(A) Co-immunoprecipitation assay. The constructs used for transfection are shown at top. COS7 cells were transfected with V5-tagged HO-1 or HO-2 expression vector and Myc-tagged PFKFB4 expression vector and then incubated for 48 $\mathrm{h}$. Whole cell extracts were used for immunoprecipitation with anti-V5 antibody, and the precipitates were subjected to Western blot analysis with anti-Myc or anti-V5 antibody. A portion of the cell lysates before immunoprecipitation was used for the confirmation of PFKFB4 protein expression in the transfected cells (Input). Then, V5-tagged HO-1 or HO-2 protein was precipitated with anti-V5 antibody. Note that PFKFB4 protein was co-precipitated with HO-1 or HO-2 protein. (B) Pull-down assay. COS7 cells were transfected with HA-tagged PFKFB4 expression vector, and cell lysates were subjected to pull-down assay with His-tagged HO-1 or HO-2 protein, lacking the TMD. Anti-HA and antiHis-tag antibodies were used for western blot analysis.

that the average body weight of male $\mathrm{HO}-2^{-/-}$mice at age of 12 weeks was heavier $(29.35 \pm 1.86 \mathrm{~g})$ than that of agematched male wild-type mice $(26.80 \pm 1.59 \mathrm{~g} ; p<0.01)$. Thus, $\mathrm{HO}-2^{-/-}$mice are obese, as reported by Sodhi et al. (2009).

It is noteworthy that male $\mathrm{HO}-2^{-/-}$mice show the decrease in mating behavior and impaired ejaculation (Burnett et al. 1998). Moreover, among the four PFKFB isozymes, only PFKFB4 is expressed in haploid spermatids (Gómez et al. 2009), in which HO-2 is abundantly expressed (Ewing and Maines 1995; Adachi et al. 2004). Considering the role of PFKFB4 in cell survival (Jeon et al. 2011; Goidts et al. 2012; Ros et al. 2012), we postulate that PFKFB4 may be responsible for the survival of haploid spermatids. In addition, the expression levels of HO-1 protein were higher in the testis and lower in the liver of HO- $2^{-/-}$mice, compared to the levels in respective organs of the wild-type mouse (Han et al. 2009, 2010). Accordingly, we analyzed the expression level of PFKFB4 protein in the testis that depends on glycolysis (Nakamura et al. 1982) and the liver that is able to release glucose. By Western blot analysis, we confirmed the identity of $\mathrm{HO}-2^{-/-}$ mice (Fig. 3A), as judged by the undetectable level of HO-2 protein in the $\mathrm{HO}_{-} 2^{--}$testis. In contrast, expression of HO-1 protein was increased in the HO- $2^{-/}$testis (Fig. 3A), as previously reported (Han et al. 2009). Importantly, there was no significant difference in the relative expression levels of PFKFB4 protein in the testis of $\mathrm{HO}^{-2^{-/}}$vs. wild type mice. The higher expression level of HO-1 in the testis may compensate for HO-2 deficiency, thereby maintaining homeostasis of spermatids. In fact, despite an increase in overall body weight in $\mathrm{HO}-2^{-/-}$mice, there was no significant difference in the testis weight between $\mathrm{HO}-2^{-/-}$mice and wild-type mice $(0.192 \pm 0.011 \mathrm{~g}$ vs. $0.205 \pm 0.162 \mathrm{~g})$. Taken together, we hypothesize that PFKFB4 may ensure the HO-2 expression in spermatids under physiological conditions and the $\mathrm{HO}-1$ expression in the $\mathrm{HO}-2^{-/-}$mouse testis.

In distinction to the testis, the expression level of HO-1 protein was lower in the liver of the HO-2 $2^{-/}$mouse (Fig. 3B), as reported previously (Han et al. 2010). Again, there was no significant difference in the PFKFB4 expression levels in the livers of $\mathrm{HO}-2^{-/-}$and wild type mice (Fig. $3 \mathrm{~B})$, which is supported in part by a recent report that hepatic cancer cells require PFKFB4 for their survival (Jeon et al. 2011). It should be noted that the liver is characterized by its capacity to synthesize and release glucose due to the presence of glucose-6-phosphatase in the hepatocyte (see Fig. 8). The lower expression level of HO-1 in liver in compensation for HO-2 deficiency may be a beneficial response for maintaining glucose homeostasis in $\mathrm{HO}-2^{-/-}$ mice that have developed hyperglycemia and insulin resis- 

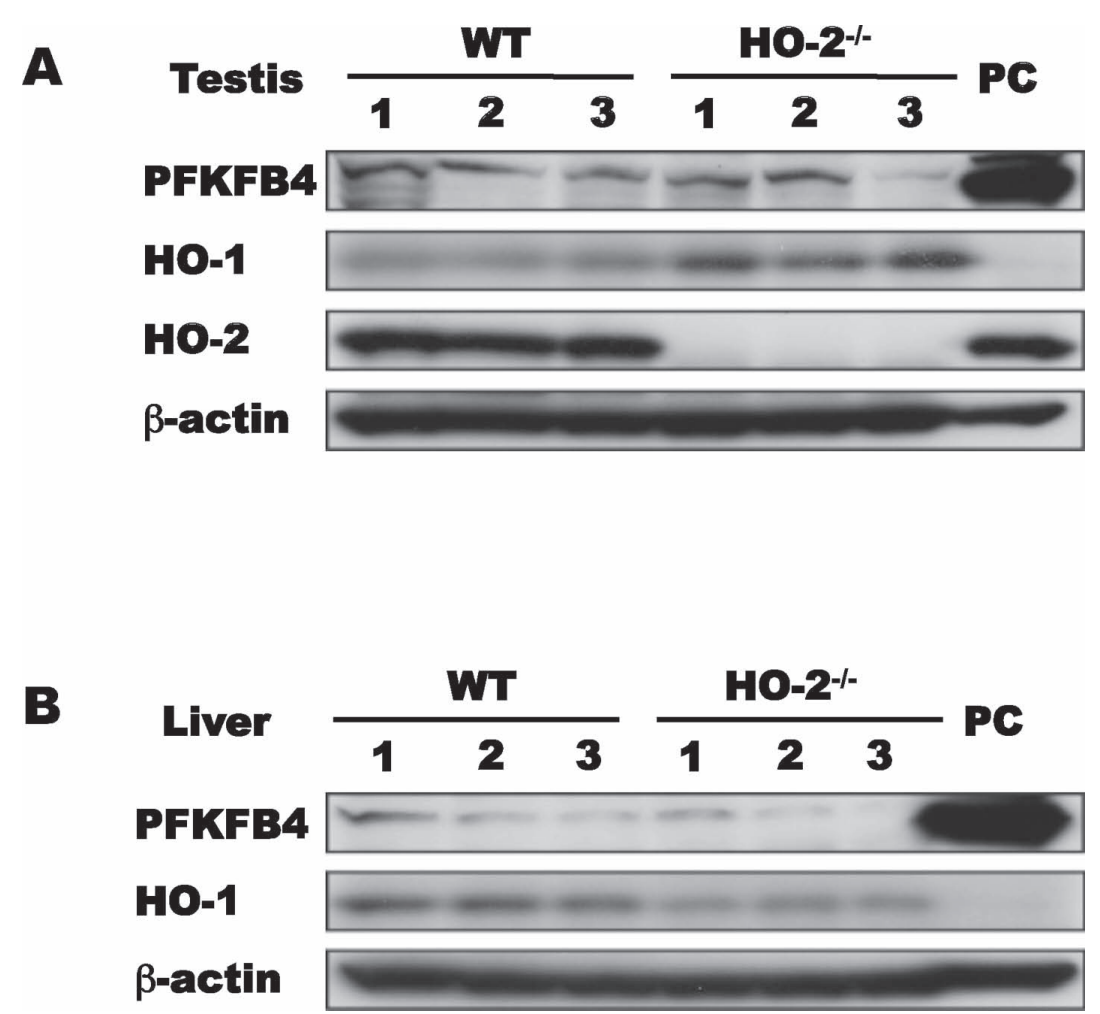

Fig. 3. Expression levels of PFKFB4 and HO-1 proteins in testis and liver of $\mathrm{HO}-2^{-/-}$mouse. The tissue extracts were prepared from the testes (A) and livers (B) of age-matched wild-type mice (10 weeks) and $\mathrm{HO}-2^{-/}$mice $(n=3$ for each group of mice), and were subjected to Western blot analysis. The data shown are derived from three animals (each animal/lane). Lane PC contained the whole cell extracts prepared from HepG2 cells that coexpressed HO-2 and PFKFB4 cDNAs as a positive control (see Fig. 5B). The relative expression level of HO-1 protein, normalized with $\beta$-actin, was increased in testis and decreased in liver of $\mathrm{HO}^{-/-}$mouse, as reported previously (Han et al. 2009, 2010). In contrast, there was no significant difference in the relative expression levels of PFKFB4 protein in the testis and liver of the wild type mouse and $\mathrm{HO}^{-/}$mouse.

tance. In this connection, heme is known to exert inhibitory effects on gluconeogenesis by down-regulating the expression of glucose-6-phosphatase (see Fig. 8) and phosphoenolpyruvate carboxykinase (Yin et al. 2007). The latter enzyme catalyzes the formation of phosphoenolpyruvate from oxaloacetate at the second step of gluconeogenesis. We postulate that the lower HO-1 expression level may lead to an accumulation of heme in hepatocytes, which in turn reduces the rate of gluconeogenesis that might have been enhanced in the $\mathrm{HO}-2^{-/-}$mouse liver, as with in diabetic patients (Meyer et al. 1998). Moreover, the down-regulation of HO-1 may decrease NADPH consumption, because heme catabolism consumes both molecular oxygen and NADPH (Tenhunen et al. 1968; Yoshida and Kikuchi 1978). NADPH is also used for regeneration of reduced glutathione that reacts with hydrogen peroxide and organic peroxides (reactive oxygen species). Thus, the distinct expression patterns of HO- 1 in liver and testis of the HO- $2^{-/-}$ mouse may reflect the adaptive responses that are beneficial for survival of the $\mathrm{HO}-2^{-/-}$mouse. In conclusion, liver metabolic features account for the inter-tissue differences in the HO-1 gene regulation.
Down-regulation of PFKFB4 in HepG2 hepatoma cells occurs concurrently with HO-1 induction under glucose deprivation conditions

To explore the regulatory link between glycolysis and heme catabolism, we analyzed short-term effects of glucose deprivation on the expression levels of PFKFB4, HO-1 and HO-2 mRNAs and proteins in HepG2 human hepatoma cells. Importantly, HepG2 cells retain the differentiated phenotype of the hepatocyte, as judged from the expression of glucokinase and glucose transporter 2 (Higuchi et al. 2011) (see Fig. 8) and a lack of ketone body utilization (Orii et al. 2008). Glucose deprivation from the culture medium may mimic hypoglycemic conditions, encountered in patients with diabetes mellitus, especially those treated with insulin. HepG2 cells are maintained in the high-glucose medium ( $25 \mathrm{mM}$ glucose); the normal plasma glucose levels are between 3.3 and $8.3 \mathrm{mM}$. Importantly, glucose deprivation induced HO-1 expression in HepG2 cells by enhancing its transcription, with the highest mRNA level achieved after $12 \mathrm{~h}$ (Chang et al. 2002), and heme inhibited gluconeogenesis in HepG2 cells (Yin et al. 2007).

For the above stated reasons, HepG2 cells were incubated for $12 \mathrm{~h}$ under glucose deprivation conditions (Fig. $4 \mathrm{~A}, \mathrm{~B})$. As expected, the expression levels of HO-1 mRNA 
$\mathbf{A}$ HepG2 (1\% FBS)

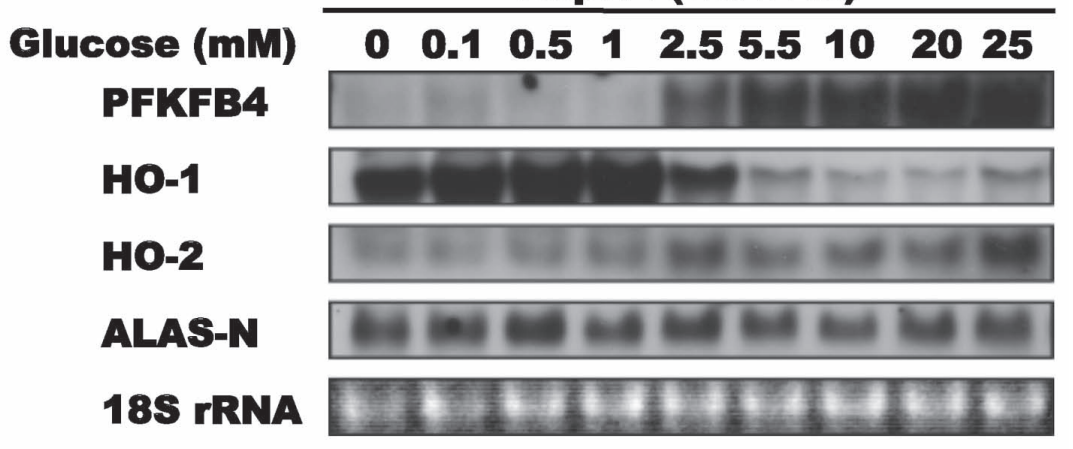

\section{B

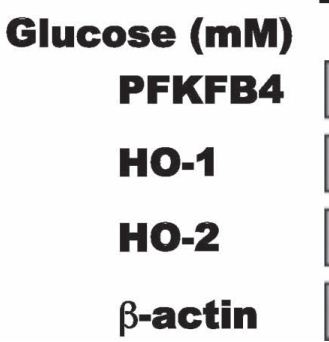

HepG2 (1\% FBS)

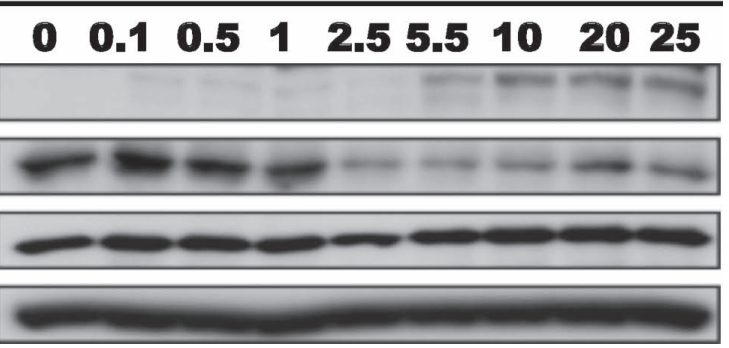

Fig. 4. Glucose deprivation downregulates PFKFB4 expression in human hepatoma cells.

HepG2 hepatoma cells were incubated for $12 \mathrm{~h}$ with the indicated glucose concentrations. (A) Northern blot analysis. Each lane contained $10 \mu \mathrm{g}$ total RNA prepared from HepG2 cells. A bottom panel shows 18S rRNA that was stained with ethidium bromide (loading control). (B) Western blot analysis. Each lane contained cell extracts (40 $\mu \mathrm{g}$ protein). A bottom panel shows $\beta$-actin as an internal control. The data shown are from one of two independent experiments with similar results.

and protein were increased in HepG2 cells at $2.5 \mathrm{mM}$ glucose and were increased further with increasing severity of glucose deprivation $(\leq 1 \mathrm{mM})$, compared to the levels under the glucose-enriched conditions $(5.5-25 \mathrm{mM})$. The plasma glucose concentration of $2.5 \mathrm{mM}$ is defined as hypoglycemia that causes hypoglycemic symptoms (Cryer 2008). In contrast to HO-1, the expression levels of PFKFB4 mRNA and protein were decreased in HepG2 cells cultured for 12 $\mathrm{h}$ under glucose deprivation $(\leq 2.5 \mathrm{mM})$. It should be noted that the expression level of HO-2 protein remained unchanged (Fig. 4B), although its mRNA level was decreased under glucose deprivation $(\leq 1 \mathrm{mM})$. Thus, $\mathrm{HO}-2$ protein seemed to be stable at least for $12 \mathrm{~h}$ under the conditions used. Moreover, the expression level of $\beta$-actin protein remained unchanged (Fig. 4B). Importantly, there was no noticeable change in the expression level of mRNA for ALAS-N, the first and the rate-limiting enzyme in heme biosynthetic pathway (Furuyama et al. 2007) (Fig. 4A). Thus, heme catabolism may be a major determinant of cellular heme contents in HepG2 cells under glucose deprivation.

In summary, under glucose deprivation conditions, PFKFB4 expression in HepG2 cells was reduced concur- rently with an induction of HO-1 expression. The remarkable induction of HO-1 expression may cause a reduction in the cellular heme concentration, which in turn increases the rate of gluconeogenesis (Yin et al. 2007). The inversely regulated expression of PFKFB4 and HO-1 may be beneficial for survival of HepG2 cells. In striking contrast, the expression level of HO-2 mRNA in HepG2 cells was decreased under glucose deprivation in parallel with PFKFB4 mRNA, but HO-2 protein level remained unchanged during the 12-h glucose deprivation. Thus, HO-2 may also contribute to the reduction of the cellular heme level.

Knockdown of HO-2 expression in HepG2 cells causes down-regulation of PFKFB4 $m R N A$ expression

The expression levels of PFKFB4 and HO-2 mRNAs were concurrently decreased in HepG2 cells under glucose deprivation, while HO-1 mRNA expression was remarkably induced (Fig. 4A). We therefore analyzed short-term consequences of HO-2 knockdown in HepG2 cells (Fig. 5A), because the knockdown of HO-2 mRNA with HO-2 siRNA (siHO-2) caused a profound decrease in HO-2 protein concurrent with an increase in the expression of HO-1 mRNA 
A

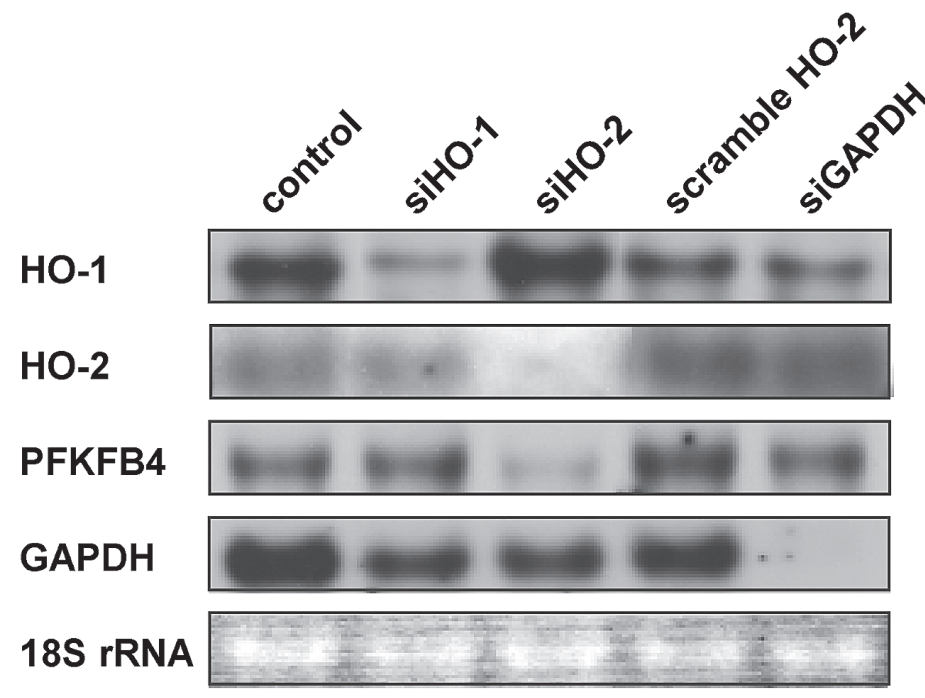

B

\begin{tabular}{|c|c|c|c|c|c|c|c|}
\hline & empty & + & + & - & - & - & - \\
\hline & $\mathrm{HO}-1$ & - & - & + & + & - & - \\
\hline & $\mathrm{HO}-2$ & - & - & - & - & + & + \\
\hline & empty & + & - & + & - & + & - \\
\hline piviyc & PFKFB4 & - & + & - & + & - & + \\
\hline
\end{tabular}

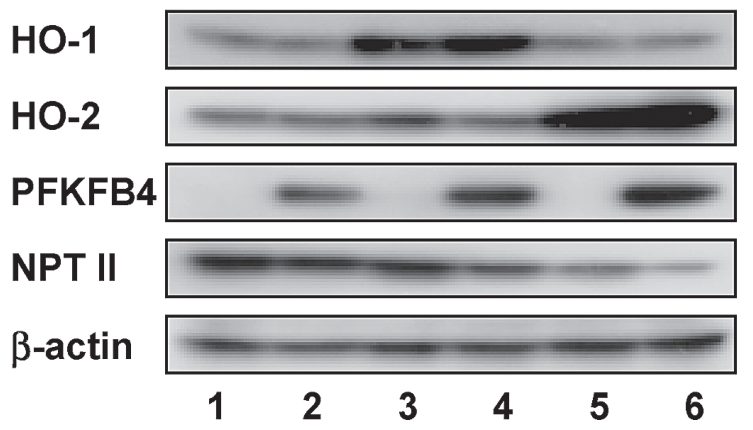

Fig. 5. Regulatory interrelationship between PFKFB4 and HO-2 in HepG2 cells.

A. Effect of HO-1 or HO-2 knockdown on the expression of PFKFB4 mRNA. HepG2 cells were treated for $36 \mathrm{~h}$ with siHO-1, siHO-2, siGAPDH, or scramble HO-2 siRNA (a negative control for siRNA). Cells were also treated with Lipofectamine RNAiMAX alone as a control. Each lane contained $10 \mu \mathrm{g}$ total RNA. A bottom panel shows 18S rRNA (loading control). Shown are representative examples of two independent experiments with similar results. Knockdown of HO-2 expression caused the expression of PFKFB4 mRNA to decrease.

B. Effect of HO-1 or HO-2 overexpression on the expression level of PFKFB4 protein. HepG2 cells were co-transfected with an expression vector coding for HO-1 or HO-2 and a Myc-PFKFB4 expression vector or an empty vector. After 48-h incubation, cells were harvested for western blot analysis. Each lane contained the same amount of total protein (20 $\mu \mathrm{g}$ protein/lane). PFKFB4 protein was detected with anti-PFKFB4 antibody. NPT II represents the expression efficiency. Shown is one representative of two independent experiments with similar results.

and protein in HepG2 cells (Ding et al. 2006). Importantly, the observed induction of HO-1 mRNA expression was in part due to the prolonged half-life of HO-1 mRNA, suggesting that HO-2 may reduce the stability of HO-1 mRNA via a hitherto unknown mechanism (Ding et al. 2006). Notably, the knockdown of HO-2 expression with siHO-2 was found to cause a reduction in expression of PFKFB4 mRNA (Fig. 5A), while the expression of HO-1 mRNA was increased, as reported previously (Ding et al. 2006). In contrast, treatment of HepG2 cells with siHO-1 or scramble HO-2 siRNA caused a marginal increase in the expression levels of HO-2 and PFKFB4 mRNAs, when normalized 
with GAPDH mRNA. Moreover, there appeared marginal increases in the expression levels of PFKFB4 and HO-2 mRNAs with siGAPDH, when normalized with stained $18 \mathrm{~S}$ rRNA. These results suggest that the expression of HO-2 and PFKFB4 mRNAs in HepG2 cells may be coordinately regulated. Taken together, we postulate that HO-2 may ensure the expression of PFKFB4 mRNA (see Fig. 8). Moreover, the expression levels of HO-1 and PFKFB4 mRNAs are inversely regulated in HepG2 cells, which is similar to their expression profiles under glucose deprivation (see Fig. 4A).

The down-regulation of PFKFB4 with HO-2 knockdown prompted us to explore a regulatory role of HO-2 in the expression of PFKFB4. Accordingly, we analyzed the effect of HO-2 overexpression on PFKFB4 expression in HepG2 cells by transient co-expression assays (Fig. 5B). The expression level of $\beta$-actin (loading control) showed the similar protein amount in each lane. The expression level of neomycin phosphotransferase (NPT) II was also measured as a control for expression efficiency of introduced vectors, showing the low transfection efficiency with HO-2 overexpression (lane 1 vs. lanes 5 and 6). Importantly, the expression levels of endogenous HO-1 and HO-2 proteins were detected in HepG2 cells (lane 1), but they remained unchanged in cells overexpressing PFKFB4 (lane 2 ), probably due to the low transfection efficiency. In contrast to the detection of endogenous $\mathrm{HO}-1$ and $\mathrm{HO}-2$, endogenous PFKFB4 was undetectable in this series of experiments (lanes 1, 3 and 5), although we detected endogenous PFKFB4 in HepG2 cells (see Fig. 4B). One obvious difference was the amounts of protein loaded to each lane (40 $\mu \mathrm{g}$ in Fig. 4B vs. $20 \mu \mathrm{g}$ in Fig. 5B). On the other hand, overexpression of c-Myc-tagged PFKFB4 caused a marginal increase in the level of co-expressed HO-1 protein (lane 3 vs. lane 4) or HO-2 protein (lane 5 vs. lane 6), when normalized with NPT II protein level. Moreover, overexpression of HO-1 or HO-2 tended to increase the level of co-expressed PFKFB4 (lane 2 vs. lane 4 or lane 6). Especially, HO-2 overexpression caused greater increases in the co-expressed PFKFB4 protein level, compared to HO-1 overexpression, when normalized with NPT II protein. Taken together with the consequences of HO-2 knockdown (Fig. 5A), we suggest that HO-2 and PFKFB4 may mutually support each expression.

\section{Over-expression of HO-2 increases the co-expressed PFKFB4 protein}

To obtain further evidence for the mutually supportive interrelationship between PFKFB4 and HO-2, we repeated the transient co-expression assays using HeLa human cervical cancer cells that are characterized by the low expression levels of endogenous HO-1 and HO-2 (Shibahara et al. 1993; Zhang et al. 2006). HeLa cells thus provide a better assay system to detect marginal effects of HO-1 or HO-2 on PFKFB4 expression. In fact, the expression of endogenous HO-1, HO-2, and PFKFB4 proteins was undetectable in the
HeLa cells transfected with a control vector and an empty vector (Fig. 6A). Importantly, the expression levels of NPT II protein (control for expression efficiency of introduced vectors) were similar in all samples, indicating that the transfection efficiency was relatively constant in transfected HeLa cells, irrespective of the vector combinations. Likewise, the expression levels of $\alpha$-tubulin (loading control) were similar in all samples. The expression level of c-Myc-tagged PFKFB4 protein was higher in cells cotransfected with HO-2 expression vector than the level in cells co-expressing HO-1, as judged with anti-PFKFB4 antibody and with anti-Myc antibody (Fig. 6A). Importantly, HO-1 overexpression did not influence the level of co-expressed PFKFB4 protein. Thus, HO-2 overexpression increased the level of co-expressed PFKFB4 protein, suggesting that HO-2 may increase the stability of PFKFB4 protein (see Fig. 8). Alternatively, considering the downregulation of PFKFB4 mRNA with HO-2 knockdown (Fig. 4A), we suggest that HO-2 may stabilize PFKFB4 mRNA. In this connection, the knockdown of HO-2 expression led to the prolongation of the half-life of HO-1 mRNA in HeLa cells (Ding et al. 2006). Lastly, we must consider another possibility that exogenous HO-2 may activate the CMV promoter that directs the expression of PFKFB4.

On the other hand, the expression level of HO-1 or HO-2 protein was higher in the HeLa cells co-transfected with the Myc-PFKFB4 vector than the level obtained with the empty vector (Fig. 6A), suggesting that overexpression of PFKFB4 may increase the levels of HO-1 and HO-2 proteins. We therefore measured the $\mathrm{HO}$ activity in the microsomal fraction of transfected HeLa cells. The HO activity was undetectable in HeLa cells cotransfected with a control vector and an empty vector or the Myc-PFKFB4 expression vector (Fig. 6B). The $\mathrm{HO}$ activity was detected in cells transfected with HO-1 or HO-2 expression vector, the activity of which was further enhanced (about twofold increase) when co-expressed with PFKFB4. Thus, PFKFB4 may increase the expression of $\mathrm{HO}-1$ and $\mathrm{HO}-2$ proteins at endoplasmic reticulum. The magnitude of the PFKFB4mediated increase in the $\mathrm{HO}$ activity was lower than that of the increase in $\mathrm{HO}$ immunoreactive protein (Fig. 6A vs. B). Such a difference may be in part due to the assay method; namely, a portion of bilirubin formed might be oxidized to biliverdin during the incubation of the reaction mixture (Baranano et al. 2002). Apparently, overexpression of PFKFB4 was associated with increased expression levels of HO-1 and HO-2 proteins. Taken together, these results suggest that PFKFB4 may stabilize HO-1 and HO-2 mRNAs or proteins. Such a regulatory interrelationship may also account for the abundant expression levels of PFKFB4 and HO-2 proteins in the mouse testis and those of PFKFB4 and HO-1 proteins in the $\mathrm{HO}-2^{-/}$mouse testis (see Fig. 3A). However, another possibility remains that c-Myc-tagged PFKFB4 may activate the CMV promoter that directs the expression of HO-1 and HO-2. 
A

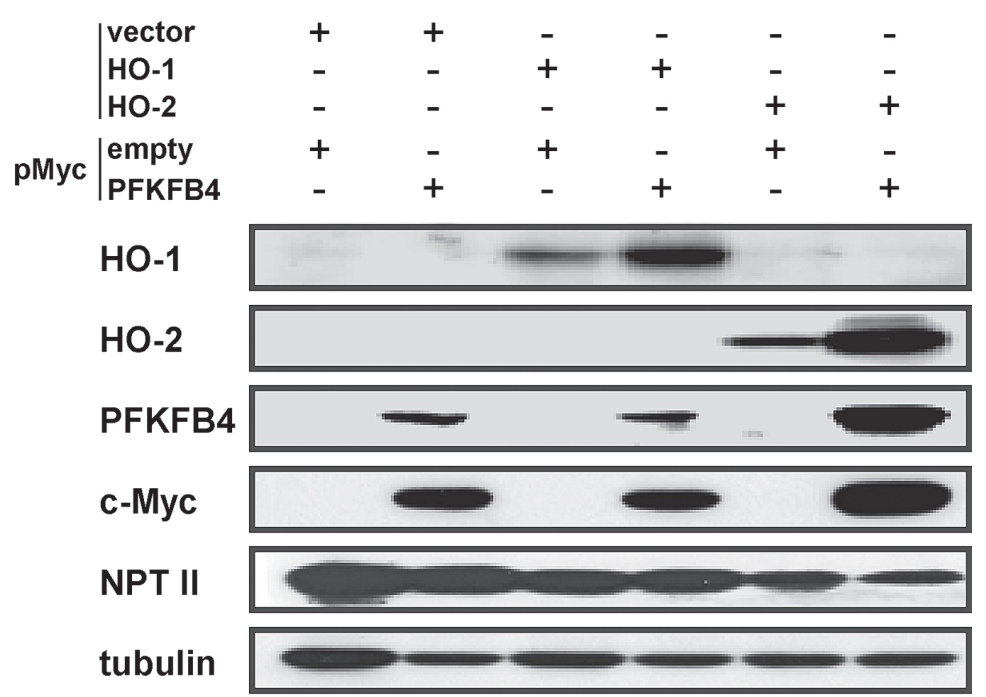

B

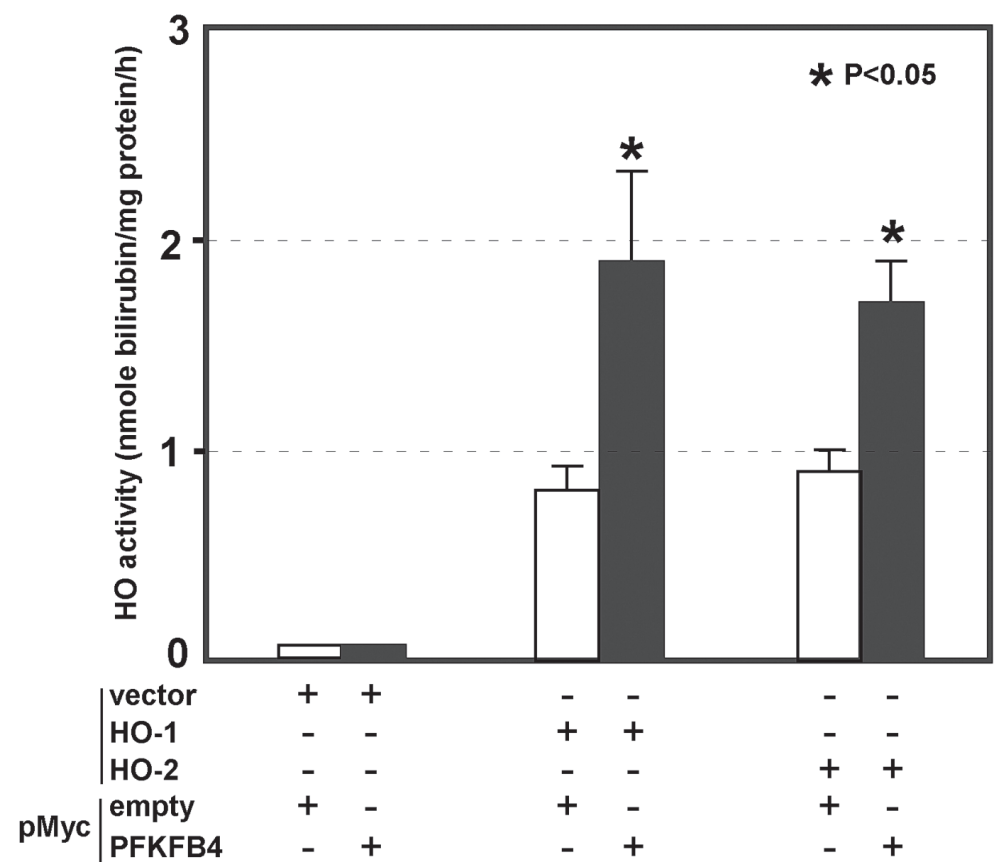

Fig. 6. HO-2 overexpression increases the co-expressed PFKFB4.

HeLa cells were co-transfected with an expression vector coding for HO-1 or HO-2 (15 $\mu \mathrm{g})$ and a Myc-PFKFB4 expression vector or an empty vector $(15 \mu \mathrm{g})$. After 24-h incubation, cells were harvested for Western blot analysis (A) and $\mathrm{HO}$ enzyme assays (B). (A) Western blot analysis of cellular extracts. Each lane contained the same amount of total protein $(20 \mu \mathrm{g}$ protein/lane). PFKFB4 protein level was measured with two kinds of antibodies: anti-PFKFB4 antibody and anti-Myc antibody (shown as c-Myc). Shown is one representative of two independent experiments with similar results. (B) HO activity in the microsomal fraction.

Down-regulation of HO-1, HO-2, and PFKFB4 mRNA expression in RPE cells under glucose deprivation conditions

To explore the metabolic implications for the glucosedeprivation-induced changes in the expression levels of PFKFB4, HO-1, and HO-2 as observed in HepG2 cells, we repeated similar experiments using human RPE cell lines as a representative model for glycolysis-dependent tissues. In fact, the retinal pigment epithelium is of the neuroectodermal origin and may depend on glycolysis, unlike the hepatocyte that is able to produce and release glucose. In addition, the retinal pigment epithelium constitutes the bloodretinal barrier that is important for survival of the photoreceptors (Davis et al. 1995; Dunn et al. 1996). Diabetes mellitus is well known to cause retinopathy that may be associated with impairment in RPE function as the 
A

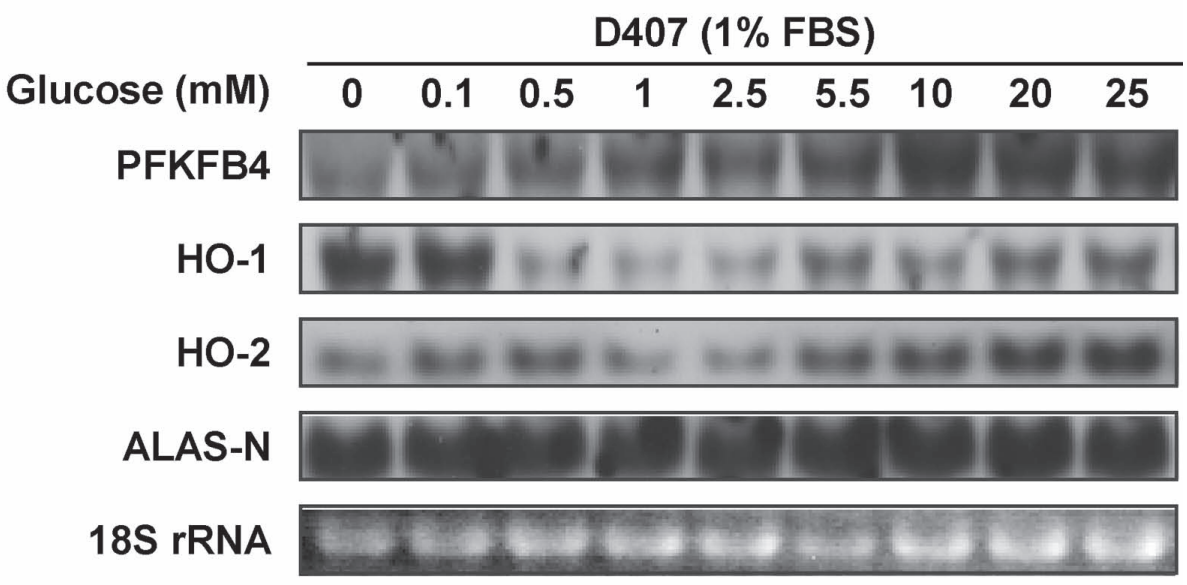

B

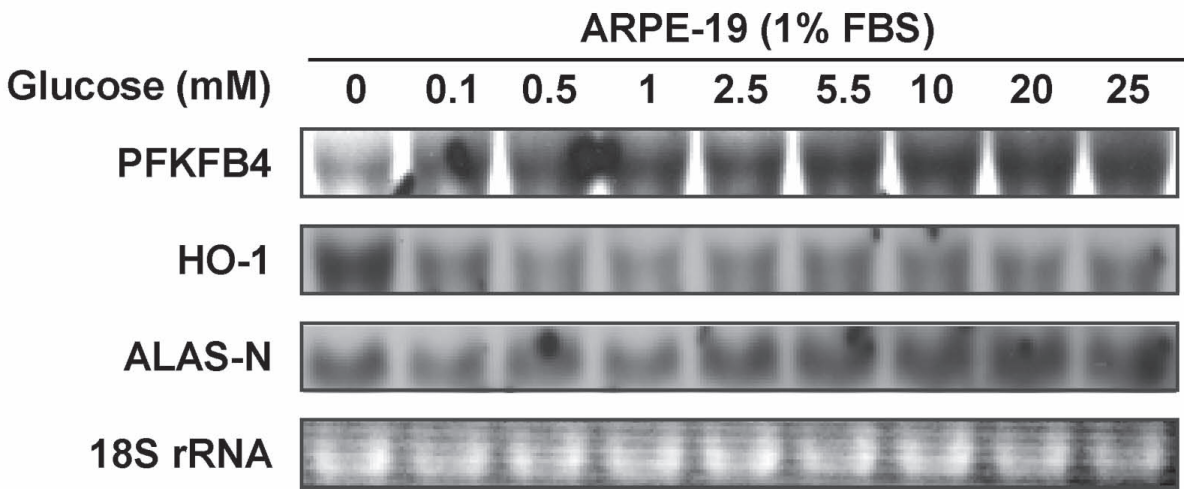

Fig. 7. Glucose deprivation down-regulates PFKFB4 expression in human RPE cells. D407 human RPE cells (A) and ARPE-19 human RPE cells (B) were incubated for $12 \mathrm{~h}$ with the indicated glucose concentrations. Each lane contained $10 \mu \mathrm{g}$ total RNA prepared from RPE cells. A bottom panel shows $18 \mathrm{~S}$ rRNA that was stained with ethidium bromide (loading control). The data shown are from one of two independent experiments with similar results.

blood-retinal barrier.

We used D407 human RPE cells to analyze the effect of glucose deprivation on the expression levels of PFKFB4, HO-1, and HO-2 mRNAs. The expression levels of PFKFB4, HO-1, and HO-2 mRNAs were decreased in D407 RPE cells under glucose deprivation (0.5 to $2.5 \mathrm{mM})$, compared to the levels with normal or higher glucose concentrations (5.5 to $25 \mathrm{mM}$ ) (Fig. 7A). The increased expression of HO- 1 mRNA at the glucose concentrations of $\leq 0.1 \mathrm{mM}$ may represent the stress response to unrealistic glucose deficiency. In fact, expression of HO-1 was increased in D407 RPE cells as well as in ARPE-19 human RPE cells in response to environmental derangements (Kuesap et al. 2008; Satarug et al. 2008). Moreover, there was no noticeable change in the expression level of ALAS-N mRNA. Thus, the expression levels of PFKFB4,
HO-1 and HO-2 mRNAs were coordinately decreased in D407 RPE cells under glucose deprivation. Importantly, the decreased expression of HO-1 mRNA in D407 cells at low glucose concentrations $(0.5$ to $2.5 \mathrm{mM})$ is in contrast to the induction of HO-1 mRNA expression observed in HepG2 cells (Fig. 7A). We also confirmed that the expression levels of PFKFB4 and HO-1 mRNAs were decreased in ARPE-19 RPE cells under glucose deprivation (Fig. 7B). Likewise, there was a marginal decrease in the expression level of ALAS-N mRNA in ARPE-19 cells, but not in D407 cells. These results suggest that the rate of glycolysis in RPE cells may be decreased in parallel with the rate of heme catabolism under glucose deprivation conditions. The decreases in the rates of glycolysis and heme catabolism may be beneficial for the survival of RPE cells under glucose deprivation. In fact, the down-regulation of HO-1 and 


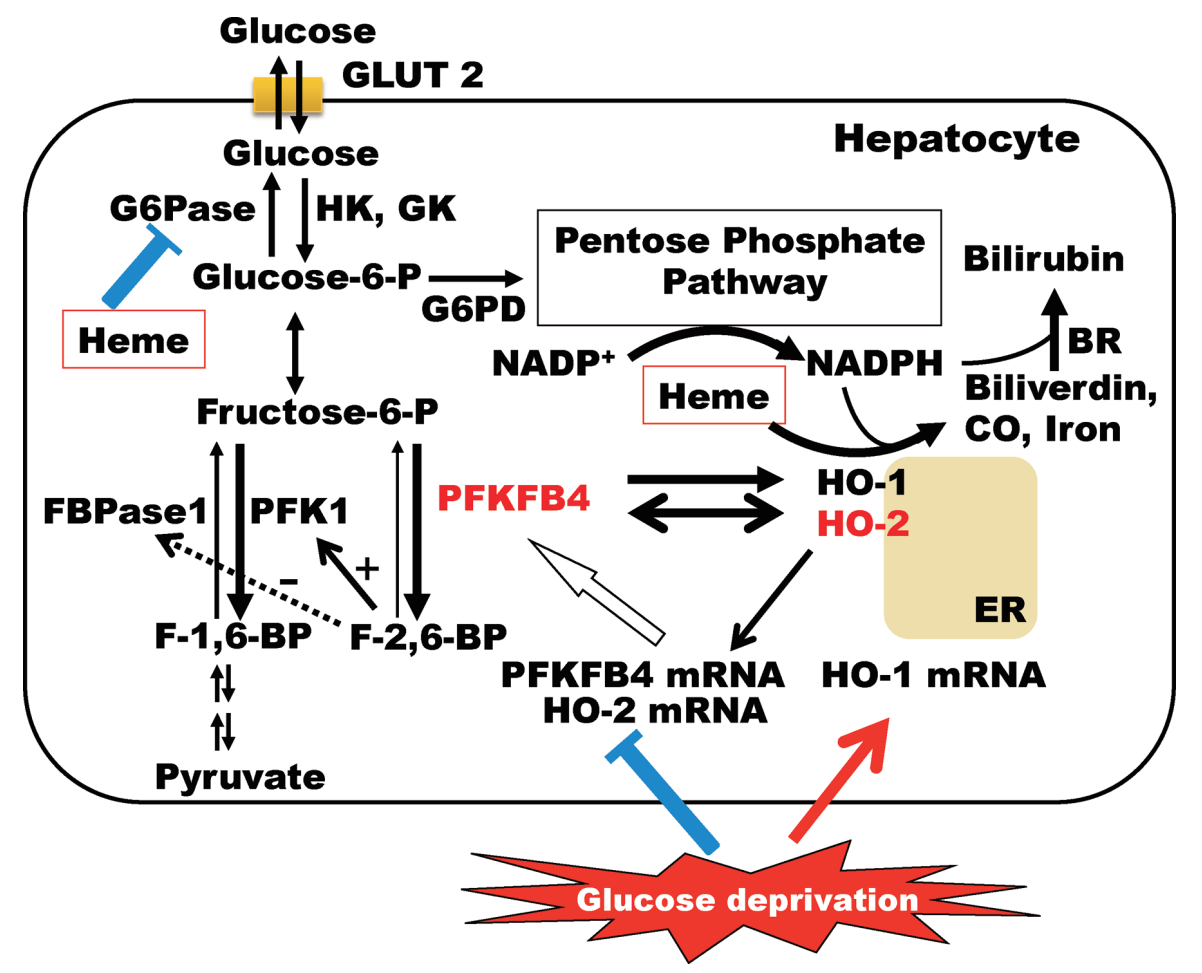

Fig. 8. Proposed regulatory network linking glycolysis with heme catabolism.

In this scheme, compartmentation is not considered, except for HO-1 and HO-2 that are anchored to endoplasmic reticulum (ER). Fructose-2,6-bisphosphate (F-2,6-BP) activates 6-phosphofructo-1-kinase (PFK1) activity and inhibits fructose-1,6-bisphosphatase (FBPase1) activity of 6-phosphofructo-1-kinase/fructose-1,6-bisphosphatase. PFKFB4 favors the synthesis of F-2,6-BP in many cell types, including hepatocytes. Glucose-6-phosphatase (G6Pase) hydrolyzes glucose-6-phosphate (Glucose-6-P) to form glucose that is released to blood. Heme inhibits G6Pase (Yin et al. 2007). Glucose-6-P is also metabolized by glucose-6-phosphate dehydrogenase (G6PD) to produce NADPH through the pentose phosphate pathway. NADPH is a cofactor that is essential for the catalytic activities of HO-1 and HO-2 as well as of biliverdin reductase (BR) (Tenhunen et al. 1968; Yoshida and Kikuchi 1978). NADPH is also consumed for regeneration of reduced glutathione that scavenges reactive oxygen species. PFKFB4 may ensure the expression of HO-1 and HO-2 (see Figs. 3 and 6). Conversely, HO-2 may ensure the expression of PFKFB4 mRNA (see Fig. 5) and protein (see Figs. 5 and 6). Glucose deprivation results in the decreased expression of HO-2 mRNA and PFKFB4 mRNA and protein, while inducing HO-1 expression.

GK, glucokinase; HK, hexokinase; GLUT2, glucose transporter 2; F-1,6-BP, fructose-1,6-bisphosphate.

HO-2 may decrease the consumption of NADPH (Fig. 8).

There are several limitations in the present study. Our proposal on the role of HO-2 in the regulation of glucose metabolism is mainly based on the analysis of gene expression, without measuring the rate of glycolysis or gluconeogenesis. In fact, the observed changes in mRNA and/or protein expression may not reflect the changes in a given enzyme activity. Further studies are required to address these issues.

\section{Concluding remarks}

The hepatocyte plays an essential role in the maintenance of the glucose homeostasis by synthesizing glucose and releasing it into the circulation (Cryer 2008). These metabolic features may account for a decrease in hepatic expression of HO-1 protein in the HO- $2^{-/-}$mouse, unlike the increased HO-1 levels in the testis and the heart (Han et al. 2009). Moreover, in HepG2 cells, the expression levels of PFKFB4 mRNA and protein were decreased under glu- cose deprivation, in which HO-1 expression was induced. Thus, glucose deprivation may blunt the rate of glycolysis while increasing the rate of heme catabolism in the HepG2 cells. In RPE cells, however, expression levels of PFKFB4, HO-1 and HO-2 mRNAs were decreased under glucose deprivation, suggesting that the rates of glycolysis and heme catabolism in RPE cells may be decreased in a coordinated manner, when glucose supply is limited. In addition, we have provided evidence that HO-2 may ensure the expression of PFKFB4. The present study thus suggests an existence of a regulatory network that maintains an appropriate balance between heme catabolism and glycolysis to meet the metabolic and physiologic function of a particular tissue.

\section{Acknowledgments}

This work was supported in part by Grant-in-Aid for Scientific Research on Priority Areas (to S.S.) from the Ministry of Education, Science, Sports, and Culture of Japan and also by 
Special Grants-in-Aid related to the East Japan Great Earthquake (to S.S.) from Astellas Foundation for Research on Metabolic Disorders and from Banyu Foundation. Bin Li was supported in part by Scholarship Sugawara Fund for the Advancement of Medical Science. We are grateful to Biomedical Research Core of Tohoku University Graduate School of Medicine for allowing us to use various facilities.

\section{Conflict of Interest}

We declare no conflict of interest.

\section{References}

Adachi, T., Ishikawa, K., Hida, W., Matsumoto, H., Masuda, T., Date, F., Ogawa, K., Takeda, K., Furuyama, K., Zhang, Y., Kitamuro, T., Ogawa, H., Maruyama, Y. \& Shibahara, S. (2004) Hypoxemia and blunted hypoxic ventilatory responses in mice lacking heme oxygenase-2. Biochem. Biophys. Res. Commun., 320, 514-522.

Baranano, D.E., Rao, M., Ferris, C.D. \& Snyder, S.H. (2002) Biliverdin reductase: a major physiologic cytoprotectant. Proc. Natl. Acad. Sci. USA, 99, 16093-16098.

Burnett, A.L., Johns, D.G., Kriegsfeld, L.J., Klein, S.L., Calvin, D.C., Demas, G.E., Schramm, L.P., Tonegawa, S., Nelson, R.J., Snyder, S.H. \& Poss, K.D. (1998) Ejaculatory abnormalities in mice with targeted disruption of the gene for heme oxygenase-2. Nat. Med., 4, 84-87.

Chang, S.H., Barbosa-Tessmann, I., Chen, C., Kilberg, M.S. \& Agarwal, A. (2002) Glucose deprivation induces heme oxygenase-1 gene expression by a pathway independent of the unfolded protein response. J. Biol. Chem., 277, 1933-1940.

Cryer, P.E. (2008) Glucose homeostasis and hypoglycemia in Williams Textbook of Endocrinology, $11^{\text {th }}$ ed., edited by H.M. Kronenberg, S. Melmed, K.S. Polonsky \& P.R. Larsen. Saunders, Elsevier, Philadelphia, PA, pp. 1503-1533.

Davis, A.A., Bernstein, P.S., Bok, D., Turner, J., Nachtigal, M. \& Hunt, R.C. (1995) A human retinal pigment epithelial cell line that retains epithelial characteristics after prolonged culture. Invest. Ophthalmol. Vis. Sci., 36, 955-964.

Ding, Y., Zhang, Y.Z., Furuyama, K., Ogawa, K., Igarashi, K. \& Shibahara, S. (2006) Down-regulation of heme oxygenase-2 is associated with the increased expression of heme oxygenase-1 in human cell lines. FEBS J., 273, 5333-5346.

Dunn, K.C., Aotaki-Keen, A.E., Putkey, F.R. \& Hjelmeland, L.M. (1996) ARPE-19, a human retinal pigment epithelial cell line with differentiated properties. Exp. Eye Res., 62, 155-169.

Ewing, J.F. \& Maines, M.D. (1995) Distribution of constitutive (HO-2) and heat-inducible (HO-1) heme oxygenase isozymes in rat testes: HO-2 displays stage-specific expression in germ cells. Endocrinology, 136, 2294-2302.

Furuyama, K., Kaneko, K. \& Vargas, P.D.V. (2007) Heme as a magnificent molecule with multiple missions: heme determines its own fate and governs cellular homeostasis. Tohoku J. Exp. Med., 213, 1-16.

Goidts, V., Bageritz, J., Puccio, L., Nakata, S., Zapatka, M., Barbus, S., Toedt, G., Campos, B., Korshunov, A., Momma, S., Van Schaftingen, E., Reifenberger, G., Herold-Mende, C., Lichter, P. \& Radlwimmer, B. (2012) RNAi screening in glioma stem-like cells identifies PFKFB4 as a key molecule important for cancer cell survival. Oncogene, 31, 3235-3243.

Gómez, M., Navarro-Sabaté, A., Manzano, A., Duran, J., Obach, M. \& Bartrons, R. (2009) Switches in 6-phosphofructo2-kinase isoenzyme expression during rat sperm maturation. Biochem. Biophys. Res. Commun., 387, 330-335.

Han, F., Takeda, K., Ishikawa, K., Ono, M., Date, F., Yokoyama, S., Furuyama, K., Shinozawa, Y., Urade, Y. \& Shibahara, S. (2009) Induction of lipocalin-type prostaglandin D synthase in mouse heart under hypoxemia. Biochem. Biophys. Res.
Commun., 385, 449-453.

Han, F., Takeda, K., Ono, M., Date, F., Ishikawa, K., Yokoyama, S., Shinozawa, Y., Furuyama, K. \& Shibahara, S. (2010) Hypoxemia induces expression of heme oxygenase-1 and heme oxygenase-2 proteins in the mouse myocardium. J. Biochem., 147, 143-151.

Hara, E., Takahashi, K., Tominaga, T., Kumabe, T., Kayama, T., Suzuki, H., Fujita, H., Yoshimoto, T., Shirato, K. \& Shibahara, S. (1996) Expression of heme oxygenase and inducible nitric oxide synthase mRNA in human brain tumors. Biochem. Biophys. Res. Commun., 224, 153-158.

Higuchi, N., Kato, M., Miyazaki, M., Tanaka, M., Kohjima, M., Ito, T., Nakamuta, M., Enjoji, M., Kotoh, K. \& Takayanagi, R. (2011) Potential role of branched-chain amino acids in glucose metabolism through the accelerated induction of the glucose-sensing apparatus in the liver. J. Cell. Biochem., 112, 30-38.

Hirai, H., Kubo, H., Yamaya, M., Nakayama, K., Numasaki, M., Kobayashi, S., Suzuki, S., Shibahara, S. \& Sasaki, H. (2003) Microsatellite polymorphism in heme oxygenase-1 gene promoter is associated with susceptibility to oxidant-induced apoptosis in lymphoblastoid cell lines. Blood, 102, 16191621.

Ishikawa, K., Takeuchi, N., Takahashi, S., Matera, K.M., Sato, M., Shibahara, S., Rousseau, D.L., Ikeda-Saito, M. \& Yoshida, T. (1995) Heme oxygenase-2. Properties of the heme complex of the purified tryptic fragment of recombinant human heme oxygenase-2. J. Biol. Chem., 270, 6345-6350.

Jeon, Y.K., Yoo, D.R., Jang, Y.H., Jang, S.Y. \& Nam, M.J. (2011) Sulforaphane induces apoptosis in human hepatic cancer cells through inhibition of 6-phosphofructo-2-kinase/fructose2,6-biphosphatase4, mediated by hypoxia inducible factor1-dependent pathway. Biochim. Biophys. Acta, 1814, 13401348 .

Kimpara, T., Takeda, A., Watanabe, K., Itoyama, Y., Ikawa, S., Watanabe, M., Arai, H., Sasaki, H., Higuchi, S., Okita, N., Takase, S., Saito, H., Takahashi, K. \& Shibahara, S. (1997) Microsatellite polymorphism in the human heme oxygenase-1 gene promoter and its application in association studies with Alzheimer and Parkinson disease. Hum. Genet., 100, 145-147.

Kitamuro, T., Takahashi, K., Ogawa, K., Udono-Fujimori, R., Takeda, K., Furuyama, K., Nakayama, M., Sun, J., Fujita, H., Hida, W., Hattori, T., Shirato, K., Igarashi, K. \& Shibahara, S. (2003) Bach1 functions as a hypoxia-inducible repressor for the heme oxygenase-1 gene in human cells. J. Biol. Chem., 278, 9125-9133.

Kuesap J., Li, B., Satarug, S., Takeda, K., Numata, I., Na-Bangchang, K. \& Shibahara, S. (2008) Prostaglandin $\mathrm{D}_{2}$ induces heme oxygenase-1 in human retinal pigment epithelial cells. Biochem. Biophys. Res. Commun., 367, 413-419.

Li, B., Takeda, K., Yokoyama, S. \& Shibahara, S. (2008) A prolylhydroxylase inhibitor, ethyl-3,4-dihydroxybenzoate, induces heme oxygenase- 1 expression in human cells through a mechanism independent of hypoxia-inducible factor- $1 \alpha . J$. Biochem., 144, 643-654.

Maines, M.D., Trakshel, G.M. \& Kutty, R.K. (1986) Characterization of two constitutive forms of rat liver microsomal heme oxygenase. Only one molecular species of the enzyme is inducible. J. Biol. Chem., 261, 411-419.

McCoubrey, W.K., Huang, T.J. \& Maines, M.D. (1997) Heme oxygenase- 2 is a hemoprotein and binds heme through heme regulatory motifs that are not involved in heme catalysis. $J$. Biol. Chem., 272, 12568-12574.

Meyer, C., Stumvoll, M., Nadkarni, V., Dostou, J., Mitrakou, A. \& Gerich, J. (1998) Abnormal renal and hepatic glucose metabolism in type 2 diabetes mellitus. J. Clin. Invest., 102, 619-624.

Miralem, T., Hu, Z., Torno, M.D., Lelli, K.M. \& Maines, M.D. (2005) Small interference RNA-mediated gene silencing of 
human biliverdin reductase, but not that of heme oxygenase-1, attenuates arsenite-mediated induction of the oxygenase and increases apoptosis in 293A kidney cells. J. Biol. Chem., 280, 17084-17092.

Nakamura, M., Fujiwara, A., Yasumasu, I., Okinaga, S. \& Arai, K. (1982) Regulation of glucose metabolism by adenine nucleotides in round spermatids from rat testes. J. Biol. Chem., 257, 13945-13950.

Nakayama, M., Takahashi, K., Kitamuro, T., Yasumoto, K., Katayose, D., Shirato, K., Fujii-Kuriyama, Y. \& Shibahara, S. (2000) Repression of heme oxygenase-1 by hypoxia in vascular endothelial cells. Biochem. Biophys. Res. Commun., 271, 665-671.

Okar, D.A., Manzano, À., Navarro-Sabatè, A., Riera, L., Bartrons, R. \& Lange, A.J. (2001) PFK-2/FBPase-2: maker and breaker of the essential biofactor fructose-2,6-bisphosphate. Trends Biochem. Sci., 26, 30-35.

Okinaga, S., Takahashi, K., Takeda, K., Yoshizawa, M., Fujita, H., Sasaki, H. \& Shibahara, S. (1996) Regulation of human heme oxygenase-1 gene expression under thermal stress. Blood, 87, 5074-5084.

Orii, K.E., Fukao, T., Song, X.Q., Mitchell, G.A. \& Kondo, N. (2008) Liver-specific silencing of the human gene encoding succinyl-CoA: 3-ketoacid CoA transferase. Tohoku J. Exp. Med., 215, 227-236.

Poss, K.D., Thomas, M.J., Ebralidze, A.K., O’Dell, T.J. \& Tonegawa, S. (1995) Hippocampal long-term potentiation is normal in heme oxygenase-2 mutant mice. Neuron, 15, 867-873.

Poss, K.D. \& Tonegawa, S. (1997) Heme oxygenase 1 is required for mammalian iron reutilization. Proc. Natl. Acad. Sci. USA, 94, 10919-10924.

Ros, S., Santos, C.R., Moco, S., Baenke, F., Kelly, G., Howell, M., Zamboni, N. \& Schulze, A. (2012) Functional metabolic screen identifies 6-phosphofructo-2-kinase/fructose-2,6-biphosphatase 4 as an important regulator of prostate cancer cell survival. Cancer Discov., http://dx.doi.org/10.1158/21598290.CD-11-0234 [Accessed: April 2, 2012].

Sakata, J., Abe, Y. \& Uyeda, K. (1991) Molecular cloning of the DNA and expression and characterization of rat testes fructose-6-phosphate, 2-kinase: fructose-2,6-bisphosphatase. $J$. Biol. Chem., 266, 15764-15770.

Satarug, S., Kikuchi, M., Wisedpanichkij, R., Li, B., Takeda, K., Na-Bangchang, K., Moore, M.R., Hirayama, K. \& Shibahara, S. (2008) Prevention of cadmium accumulation in retinal pigment epithelium with manganese and zinc. Exp. Eye Res., 87, 587-593.

Shibahara, S. (2003) The heme oxygenase dilemma in cellular homeostasis: new insights for the feedback regulation of heme catabolism. Tohoku J. Exp. Med., 200, 167-186.

Shibahara, S., Han, F., Li, B. \& Takeda, K. (2007) Hypoxia and heme oxygenases: oxygen sensing and regulation of expression. Antiox. Redox Signal., 9, 2209-2225.

Shibahara, S., Muller, R., Taguchi, H. \& Yoshida, T. (1985) Cloning and expression of cDNA for rat heme oxygenase. Proc. Natl. Acad. Sci. USA, 82, 7865-7869.

Shibahara, S., Yoshida, T. \& Kikuchi, G. (1978) Induction of heme oxygenase by hemin in cultured pig alveolar macrophages. Arch. Biochem. Biophys., 188, 243-250.

Shibahara, S., Yoshizawa, M., Suzuki, H., Takeda, K., Meguro, K. \& Endo, K. (1993) Functional analysis of cDNAs for two types of human heme oxygenase and evidence for their separate regulation. J. Biochem. (Tokyo), 113, 214-218.

Sodhi, K., Inoue, K., Gotlinger, K.H., Canestraro, M., Vanella, L., Kim, D.H., Manthati, V.L., Koduru, S.R., Falck, J.R.,
Schwartzman, M.L. \& Abraham, N.G. (2009) Epoxyeicosatrienoic acid agonist rescues the metabolic syndrome phenotype of HO-2-null mice. J. Pharmacol. Exp. Ther., 331, 906-916.

Sun, D., Sato, M., Yoshida, T., Shimizu, H., Miyatake, H., Adachi, S., Shiro, Y. \& Kikuchi, A. (2000) Crystallization and preliminary X-ray diffraction analysis of a rat biliverdin reductase. Acta Crystallogr. D Biol. Crystallogr., 56 (Pt 9), 1180-1182.

Takahashi, K., Hara, E., Suzuki, H., Sasano, H. \& Shibahara, S. (1996) Expression of heme oxygenase isozyme mRNAs in the human brain and induction of heme oxygenase- 1 by nitric oxide donors. J. Neurochem., 67, 482-489.

Takahashi, K., Nakayama, M., Takeda, K., Fujita, H. \& Shibahara, S. (1999) Suppression of heme oxygenase-1 mRNA expression by interferon- $\gamma$ in human glioblastoma cells. J. Neurochem., 72, 2356-2361.

Takeda, K., Ishizawa, S., Sato, M., Yoshida, T. \& Shibahara, S. (1994) Identification of a cis-acting element that is responsible for cadmium-mediated induction of the human heme oxygenase gene. J. Biol. Chem., 269, 22858-22867.

Tenhunen, R., Marver, H.S. \& Schmid, R. (1968) The enzymatic conversion of heme to bilirubin by microsomal heme oxygenase. Proc. Natl. Acad. Sci. USA, 61, 748-755.

Udono-Fujimori, R., Takahashi, K., Takeda, K., Furuyama, K., Kaneko, K., Takahashi, S., Tamai, M. \& Shibahara, S. (2004) Expression of heme oxygenase- 1 is repressed by interferon- $\gamma$ and induced by hypoxia in human retinal pigment epithelial cells. Eur. J. Biochem., 271, 3076-3084.

Yamada, N., Yamaya, M., Okinaga, S., Nakayama, K., Sekizawa, K., Shibahara, S. \& Sasaki, H. (2000) Microsatellite polymorphism in the heme oxygenase-1 gene promoter is associated with susceptibility to emphysema. Am. J. Hum. Genet., 66, 187-195.

Yalcin, A., Clem, B.F., Simmons, A., Lane, A., Nelson, K., Clem, A.L., Brock, E., Siow, D., Wattenberg, B., Telang, S. \& Chesney, J. (2009) Nuclear targeting of 6-phosphofructo2-kinase (PFKFB3) increases proliferation via cyclin-dependent kinases. J. Biol. Chem., 284, 24223-24232.

Yasumoto, K., Takeda, K., Saito, H., Watanabe, K., Takahashi, K. \& Shibahara, S. (2002) Microphthalmia-associated transcription factor interacts with LEF-1, a mediator of Wnt signaling. EMBO J., 21, 2703-2714.

Yin, L., Wu, N., Curtin, J.C., Qatanani, M., Szwergold, N.R., Reid, R.A., Waitt, G.M., Parks, D.J., Pearce, K.H., Wisely, G.B. \& Lazar, M.A. (2007) Rev-erb $\alpha$, a heme sensor that coordinates metabolic and circadian pathways. Science, 318, 1786-1789.

Yoshida, T., Biro, P., Cohen, T., Muller, R.M. \& Shibahara, S. (1988) Human heme oxygenase cDNA and induction of its mRNA by hemin. Eur. J. Biochem., 171, 457-461.

Yoshida, T., Ishikawa, K. \& Sato, M. (1991) Degradation of heme by a soluble peptide of heme oxygenase obtained from rat liver microsomes by mild trypsinization. Eur. J. Biochem., 199, 729-733.

Yoshida, T. \& Kikuchi, G. (1978) Purification and properties of heme oxygenase from pig spleen microsomes. J. Biol. Chem., 253, 4230-4236.

Yoshida, T. \& Sato, M. (1989) Posttranslational and direct integration of heme oxygenase into microsomes. Biochem. Biophys. Res. Commun., 163, 1086-1092.

Zhang, Y., Furuyama, K., Kaneko, K., Ding, Y., Ogawa, K., Yoshizawa, M., Kawamura, M., Takeda, K., Yoshida, T. \& Shibahara, S. (2006) Hypoxia reduces the expression of heme oxygenase- 2 in various types of human cell lines. A possible strategy for the maintenance of intracellular heme level. FEBS $J ., 273,3136-3147$. 